\title{
LA POLÍTICA DE LOS JÓVENES: ENTRE NUEVOS MEDIOS Y ESPACIOS PÚBLICOS
}

\section{YOUNG PEOPLE'S POLITICS: BETWEEN NEW MEDIA AND PUBLIC SPACE}

\author{
Rolando Marini \\ Università per Stranieri di Perugia. Perugia/Italia \\ rolando.marini@unistrapg.it
}

Recibido/Received: 15/06/2018

Modificado/Modified: 01/09/2018

Aceptado/Accepted: 22/09/2018

\section{RESUMEN}

En este trabajo se presentan los resultados de una investigación que pretende reconstruir la relación entre jóvenes italianos, uso de los medios y espacio público. El objetivo es intentar superar los límites del concepto de participación política, tomando como punto de referencia los conceptos-modelos de self-actualizing citizen y el de culturas cívicas. A través de 45 entrevistas a jóvenes de entre 19 y 25 años y según un enfoque cualitativo, analizamos las estrategias realizadas en los entornos comunicativos múltiples: los medios tradicionales y digitales, las relaciones entre pares. El uso de la información y la gestión de las plataformas sociales nos permiten reconstruir una tipología que nos enseña la importancia de la selección. Destacamos dos principales tipologías de "activismo": compromiso informativo y activismo discursivo. Investigamos algunas dinámicas de liderazgo de opinión, tanto interpersonales como mediales. Por último, analizamos las experiencias de militancia, de las que brindamos la reconstrucción reflexiva por parte de los jóvenes entrevistados.

\section{PALABRAS CLAVE}

Nuevos medios; influencia; usos de los medios; implicación cívica; ciudadanía.

\section{SUMARIO}

1. Dos propuestas para dos problemas metodológicos. 2. El diseño de la investigación y las técnicas de recogida de datos. 3. Los jóvenes italianos entre viejos y nuevos medios. 4. Activismo discursivo y liderazgo de opinión. 5. Implicación y militancia. 6. Conclusiones. Bibliografía.

\begin{abstract}
This paper presents the main results of a survey regarding the relationship among Italian young people, media uses and the public space. An effort is conducted to overtake the limits of the concept of political participation, looking at the concepts-models of self-actualizing citizen and the one of civic cultures. Through 45 in-depth interviews to 19-25 years old Italian people, according to a qualitative approach, the survey analyzes the strategies young people adopt within multiple communication environments: traditional and digital media; peer relationships. The information use and the social platforms management allow building a typology that shows the importance of selectivity also for its impact on civic engagement. Two principal forms of pre-political engagement emerge, related to news use and to discursive interchange. The survey investigates some opinion leadership dynamics, both interpersonal
\end{abstract}


and media-related. At last some militancy experiences are analyzed, offering the reflexive reconstruction by the interviewees.

\section{KEYWORDS}

New Media; Influence; Media Uses; Civic Engagement; Citizenship.

\section{CONTENTS}

1. Two proposals for two methodological problems. 2. The survey design and the techniques for data collection. 3. Italian young people between old and new media. 4. Discursive activism and opinion leadership. 5. Engagement and militancy. 6. Conclusions. References.

\section{DOS PROPUESTAS PARA DOS PROBLEMAS METODOLÓGICOS}

El estudioso que lleve a cabo investigaciones acerca de la relación entre cultura juvenil, participación política y nuevos medios se encuentra sumergido en un contexto científico repleto de problemas teóricos y metodológicos para los cuales es necesario elegir soluciones. Estos nudos problemáticos se refieren básicamente al concepto de participación política y a la visión dominante del papel que desempeñan las nuevas tecnologías de la comunicación en la sociedad contemporánea: problemas que, presentes a nivel general, se hacen más evidentes a la hora de estudiar el mundo de los jóvenes. De hecho, la inclinación hacia la participación política por parte de los jóvenes se asocia sin duda a la inclinación hacia la relacionalidad mediada de los entornos digitales. Sin embargo, para algunos científicos sociales esta nueva tipología de mediatización constituiría una fuerza social positiva y para otros, en cambio, una fuerza negativa e invertida con respecto a las potencialidades participativas; en este último caso, en sinergia con otros factores de desintegración social (según el modelo teórico de Putnam, 2001). De ahí que parezca necesario intentar revisar ante todo estos dos nudos teórico-metodológicos.

En los debates sociológicos internacional e italiano se ha mantenido hasta hoy una acepción más bien normativa del concepto de participación política, referido - directamente o indirectamente - no solo al modelo del ciudadano consciente, propio de la tradición liberaldemocrática, sino también al modelo del (joven) militante, protagonista de los movimientos de protesta, en otras palabras un modelo de acción colectiva como expresión de tensión radical al cambio; de ahí que resulte imposible entender las raíces de la evolución que se está dando y la naturaleza de la reconfiguración de la política (ya destacada en otros estudios acerca de los movimientos: Melucci, 1982 y 1989). Por lo tanto, el problema se refiere a un concepto de participación - que no solo es política sino también ampliamente cívica (Schudson, 1998 y Dahlgren, 2009) - que siga incorporando una serie de actitudes esperadas (especialmente si se habla de los jóvenes), muchas veces de índole antagonístico, como el boicoteo (Bennett 1998), quizás relacionadas con las nuevas formas de conectividad mediada (Bennett 2008a). En este punto se ha formado un frente crítico bastante amplio, a veces incierto, que no parece tener éxito para afirmarse como prevalente (Smith et al. 2005; Benedicto y Morán 2007; Harris; Wynn y Younes 2010; Morán y Benedicto, 2016).

Desplazar el enfoque desde la participación política a la "implicación cívica" nos ha permitido abrir un debate mucho más amplio y consciente acerca de las transformaciones realizadas (Dahlgren, 2009; Bartoletti y Faccioli, 2013). Sin embargo, sigue habiendo una división entre dos enfoques: uno que privilegia el desarrollo y la "visibilidad" efectiva de formas colectivas de acción política; y el otro que privilegia el desplazamiento de las culturas 
políticas mucho más allá de las fronteras convencionales.

En este sentido, podría ser útil tomar en consideración el modelo del ciudadano propuesto por Bennett para clasificar las distintas formas de ciudadanía que están surgiendo entre las generaciones más jóvenes, el del self-actualizing citizen, opuesto en sentido ideal-típico al modelo tradicional del dutiful citizen. En esta figura de ciudadano desaparecen la pertenencia a un partido o sindicato, la atención constante y responsable a los eventos de la política de las instituciones centrales a través de medios de información mainstream; se consolida una política trasladada a la dimensión privada o local, propensa al voluntariado social, y fomentada por una conectividad digital (Bennett 2008b: 14). Sin embargo, el modelo parece contar con notables potencialidades metodológicas, sobre todo gracias a la idea de un proceso de actualización personal de la relación con la política, abierto a caminos exploratorios y a implicaciones intermitentes, desplazando claramente su baricentro hacia la sociedad civil.

Dahlgren (2005 y 2009) abre una vía metodológica alternativa a la puramente normativa; el autor propone el concepto de "culturas cívicas" como marco para el análisis de la manera en la que las personas se convierten en "ciudadanos-agentes", señalando la importancia fundamental de la "dimensión procesual y del contexto" de este marco (2005: 158). Las culturas cívicas no se concretizan en prácticas formalizadas, sino más bien encuentran en la interacción social los contextos de su propio desarrollo, asumiendo un carácter pre-político o incluso no político, carácter que define las potencialidades de la participación (Ibíd.). Dahlgren subraya la necesidad de las culturas cívicas de instalarse en el dado por descontado y, de acuerdo con Habermas, señala que deberían residir en los mundos vitales (2013, p. 31). Una orientación parecida, atenta al proceso y al aprendizaje, también se ha visto reflejada en algunos estudios españoles (Benedicto y Morán, 2003 y 2007; Morales, 2005 y 2014; Morán y Benedicto, 2016) e italianos (Caniglia, 2002).

De ahí que pudiera plantearse la posibilidad de fundir los enfoques de Bennett y Dahlgren e investigar la procesualidad no lineal de una "apropiación negocial" de la política por parte de los jóvenes. Todo esto se complementaría con el problema de la suspensión de la identidad, típica de los caminos exploratorios socio-culturales de la indefinición juvenil (Erikson, 1959 y 1968; Caniglia, 2002). Si la implicación cívica se cruza con los procesos de identificación, no podemos ignorar el desarrollo de las habilidades emotivas y relacionales de los jóvenes como contexto para las habilidades cívicas y políticas o pre-políticas (Morán y Benedicto, 2016; Serpieri y Tota, 2018).

El estudio que presentamos a continuación se propone, según estas líneas, investigar la relación de los jóvenes no tanto con la política entendida en sentido institucional, sino más bien con el espacio público, es decir con los espacios múltiples donde se construyen y vehiculan los debates alrededor de los temas relacionados con la vida social. Esta perspectiva nos permite tomar en consideración formas "participativas" en un espacio público que se ha alargado y diferenciado, ya que acoge a distintos actores y temas, lenguajes y códigos que antes no vivían en él (Dahlgren, 2005; Sorrentino, 2008; Marini ,2011). En este sentido, cabe destacar la propuesta teórica de Schudson (1998) que habla de "ciudadano monitorizante" refiriéndose a las estrategias de la nueva participación en un entorno más complejo de la vida pública, basadas en una atención inconstante pero no distanciada.

Al lado de los conceptos de implicación y de ciudadanía, se propone en este caso otro nudo problemático - pero estrechamente relacionado con los anteriores - relativo al papel de las tecnologías digitales y sobre todo de los medios sociales en relación con los jóvenes y con las formas de participación cívica (Bennett, 2008a; Lovink, 2011; Sunstein, 2017). En este ámbito notamos una proliferación de teorías $\mathrm{y}$ de estudios que proponen las nuevas tecnologías de la comunicación desde una perspectiva casi mítica, principalmente conciernen 
la relación entre los jóvenes y las tecnologías digitales (Twenge, 2006; Watkins, 2009).

De todas formas y más allá de algunos extremismos, no podemos excluir que gracias a la Red se ha engendrado, a través de las conexiones digitales de las personas y de sus vidas, no solo otro espacio de mediación, sino también un territorio real de producción tanto de las relaciones sociales como de significados, incluso con valor político (Boccia Artieri, 2016).

El objetivo principal de la investigación que presentamos a continuación es el de incluir estos caminos de orientación dentro de un ecosistema relacional y medial múltiple, a partir del cual los sujetos pueden sacar recursos comunicativos y cognitivos que vuelven a organizar con elaboraciones personales, aunque nunca desconectadas de los contextos socioculturales de pertenencia. No se trata de un problema nuevo: el tratamiento de la información y la construcción de los significados constituyen un proceso en el que la interacción con una gran variedad de entornos comunicativos les permite a las personas construir significados y marcos constantemente re-negociados con los demás y con los textos (Gamson, 1992; Neuman; Just y Crigler, 1992; Livingstone, 1999).

Como consecuencia, la perspectiva de este trabajo hace referencia a la visión hermenéutica de la relación entre medios de comunicación, relaciones sociales y procesos de interpretación: perspectiva que pretende entender el maridaje y la sinergia entre universos relacionales "mediados" y universos relacionales no mediados, en la lucha entre global y local (Thompson. 1995). Dentro de estas dos intersecciones fundamentales, las personas recorren caminos de construcción de la identidad y de orientación en el espacio público que implican la gestión de habilidades comunicativas complejas y el uso de distintos recursos cognitivos y emocionales, mediales y no mediales. Por lo tanto, resulta imprescindible destacar la manera en la que los entornos comunicativos brindados por las tecnologías digitales se proponen como medios para la gestión del self (Stella et al. 2014; Boccia, Artieri et al. 2017), relacionándose con los lugares y los espacios que cooperan en el proyecto de identidad.

\section{EL DISEÑO DE LA INVESTIGACIÓN Y LA TÉCNICA DE RECOGIDA DE DATOS}

A partir de este marco teórico de referencia, se origina la necesidad de analizar la relación entre los jóvenes y los distintos entornos comunicativos en los que se divide y adquiere sentido su socialidad. De entre todos estos entornos comunicativos múltiples, el presente estudio analiza los de tipo offline y online; más en detalle, pretende entender cuáles podrían ser las modalidades o, si queremos, las estrategias más o menos conscientes de gestión de este mosaico de oportunidades. Por lo tanto, hemos intentado tomar en consideración los entornos familiar, amistoso y local-territorial, en su intersección con todos los medios de comunicación y los entornos virtuales.

La investigación presenta un diseño que se propone analizar las siguientes dimensiones:

a) la constante interacción entre las actividades y las relaciones online y offline;

b) los consumos mediales y de información en relación a las prácticas personales y colectivas (aspecto experiencial y auto-reflexivo);

c) las experiencias de militancia o voluntariado;

d) el interés hacia temáticas y/o eventos de relevancia pública;

e) la relación entre este interés y la vida privada;

f) la costumbre de tratar problemas sociales y políticos;

g) los procesos de liderazgo de opinión; 
h) la evaluación de la experiencia de implicación o de participación.

La técnica de recogida de datos que mejor podía reflejar esta visión del método era la entrevista a fondo, una tipología de entrevista que nos permite construir un diálogo con caminos cada vez más distintos. Las entrevistas cualitativas (o no estandarizadas) implican una adaptación a las argumentaciones personales, que enriquece la calidad de la información, recuperando la relación entre reconstrucción de los elementos de hecho e interpretación, y fomentando aspectos de la reflexividad relacionados incluso con fragmentos biográficos (Schwartz y Jacobs, 1979; Montesperelli, 1998; Losito, 2004; Addeo y Montesperelli, 2007).

La muestra que hemos construido es de tipo no probabilístico; su selección se ha orientado hacia algunos objetivos: el equilibrio entre géneros, la comparación entre metrópoli (Roma) y ciudad media (Perusa y Ascoli Piceno) y entre estudiantes y no estudiantes. De entre todos los estudiantes (universitarios), hemos entrevistado a los que están cursando estudios humanístico-sociales y científico-técnicos; tanto estudiantes que residen en estas ciudades, como estudiantes que viven fuera. Estos últimos nos han llevado a una diversificación muy amplia de los entornos socio-territoriales de procedencia.

Hemos llevado a cabo 45 entrevistas: el bloque más consistente (32 entrevistas) desde mayo de 2015 hasta marzo de 2016, y las otras realizadas entre noviembre de 2016 y junio de 2018. La Tabla 1 recoge los valores principales y las características de la muestra analizada.

Tabla 1: Composición de la muestra (por género, domicilio y condición profesional de los entrevistados)

\begin{tabular}{|c|c|c|c|c|c|c|}
\hline Género & Perusa & Roma & Ascoli Piceno & Estudiante & Trabajador & Total \\
\hline Hombres & 16 & 4 & 3 & 18 & 5 & 23 \\
\hline Mujeres & 14 & 6 & 2 & 17 & 5 & 22 \\
\hline
\end{tabular}

Fuente: elaboración propia

En el texto que presentamos a continuación, se proponen fragmentos de entrevistas que parecen emblemáticos de una posición o de una representación del problema analizado, dejándolos en su forma original, para cumplir (aunque entre los límites de la traducción) con las pautas de la expresión "natural".

\section{LOS JÓVENES ITALIANOS ENTRE VIEJOS Y NUEVOS MEDIOS}

En la presente investigación, las estrategias de acceso a los discursos públicos y a la narración de los eventos adquieren una importancia central: por lo tanto, el uso de los medios como recurso para informarse es un objeto que juega un papel de relieve en sí mismo, es decir constituye de por sí una forma primaria de participación en el espacio público. El "juego de interacción", que a menudo solemos encontrar en los medios para informarse, destaca el carácter "social" de las plataformas, y el impacto en el proceso de adquisición y elaboración de la información, esencialmente la capacidad atribuida a las tecnologías de la comunicación para establecer las pautas y los rituales de la interacción social (Gauntiett, 2000; Boccia Artieri, et al. 2017). Por este motivo, cualquiera investigación que abarque el tema de los jóvenes y del espacio público debería pretender detectar e interpretar las 
incongruencias, las especificidades disonantes de los caminos individuales. De ahí que hemos conseguido agrupar una constelación de configuraciones no alienadas ni con la presunta "mayoría" ni con la ideología del medio.

Las entrevistas llevadas a cabo destacan con claridad que los jóvenes son principalmente usuarios de Internet, con modalidades de uso enfocadas al intercambio frecuente a través de las plataformas sociales (la más utilizada es Facebook). Sin embargo, las dietas mediales personales constituyen una mezcla muy variable de caso a caso, que implica otros medios, sobre todo la televisión y, a seguir, los periódicos en papel y en línea. Además, cabe señalar que el uso de los medios, tanto para diversión como para información, suele parecer muy a menudo como un proceso en el que se definen los cambios que caracterizan niveles y etapas del camino biográfico, implicando principalmente momentos de revisión crítica de la relación con los medios sociales y un re-diseño de esta misma mezcla.

En las evaluaciones recogidas, la mayoría de nuestros entrevistados pone de relieve las razones que están en la base de sus preferencias para las nuevas formas de consumo medial:

a) la adhesión a la idea del acceso libre y gratuito a los contenidos culturales e informativos;

b) la búsqueda de un entorno para el uso y la conexión que represente y permita fomentar una identidad colectiva exclusiva, en la que ejercer competencias tecnológicas específicas y de conectividad extendida.

En este marco, destacan importantes diferencias, resaltadas en el uso de Facebook, la plataforma en la que la totalidad de los entrevistados declara tener su perfil desde hacía años. Las modalidades de uso pueden variar en función de: a) la frecuencia de las actividades; b) la gestión del network. A partir de estas dos dimensiones, ha sido posible construir una tipología, con dos individuos que reunían características opuestas en sentido ideal típico. Los que utilizan mucho la plataforma tienden a alargar y no seleccionar el network virtual (que podríamos llamar "acogedores"); los que utilizan la plataforma para fines específicos tienden a ser selectivos en la gestión del network "amistoso" de la plataforma ("selectivos"). Los primeros parecen propensos a valorizar un ego dilatado aunque superficial, juguetón y ritualizado según las pautas y las sanciones del like. Los selectivos son más prudentes y no se dejan someter, sino más bien rehúyen de la "popularidad" que la web social podría devolverles. En línea general, interactúan casi exclusivamente - aunque no necesariamente con los que suelen encontrar offline (los amigos íntimos, los compañeros de la universidad o de trabajo), es decir con quien ya tienen afinidad y en los que confían. Para muchos de estos entrevistados se trata de una adquisición que se ha dado a lo largo de una reconsideración crítica hacia el carácter "demasiado" abierto y entrometido de la plataforma. Cabe señalar, como ejemplo, la declaración de Intal, estudiante de comunicación de 21 años de Perusa:

Tengo 24 amigos en Facebook porque no quiero compartir las frases o las fotos que más me gustan. Se trata de mis amigos verdaderos (...). Solo amigos reales, no acepto a personas que no conozco: los mejores amigos y algunos compañeros de la universidad. Porque Facebook es extremadamente personal (...). He vuelto a crear mi perfil de Facebook precisamente para eliminar de mi vida algunos antiguos amigos míos, porque a mi el hecho de que conoces a Fulanito y a Menganito no me gusta (...).

Otros entrevistados que pertenecen a este grupo disminuyen el carácter de intimación de la plataforma a favor da una conectividad extendida, limitándose su uso a pocas funcionalidades. Asimismo, Into40 manifiesta su molestia por la proliferación, en los intercambios en Facebook, de futilidad y polémica fines a sí mismas, que gestiona con una actitud de escape. Into39, más que alejarse, opta por una estrategia de alta selectividad de los 
argumentos y de los interlocutores.

La selectividad abre paso al problema de la relación con la multimedialidad y el de las competencias cross-mediales (Boccia Artieri ,2016). La centralidad de Internet se cruza con las distintas formas de integración y de hibridación con otros medios (Bolter y Grusin, 1999; Chadwick, 2013), no solo desde el punto de vista productivo, sino también desde el del uso. De hecho, los contenidos de muchos de nuestros jóvenes usuarios parecen pertenecer a un contexto medial único e indistinto, a un medium que podemos definir supermedium.

Bajo esta perspectiva, podría ser útil analizar el consumo de la televisión. Las entrevistas recogidas ponen en relieve que más de la mitad de los entrevistados declara no mirar normalmente la televisión. Las numerosas respuestas negativas a la pregunta sobre el uso de la televisión esconden una entramada red de contradicciones: de hecho, gracias a las otras preguntas, los entrevistados han podido asociar con facilidad determinadas costumbres al consumo de géneros televisivos y de programas específicos. Esta ambigüedad parece ante todo atestiguar un desprendimiento entre la reputación del medio y el sentido compartido de una determinada identidad digital entre los jóvenes. Sin embargo, debería considerarse como el resultado de un consumo de los contenidos televisivos desinstitucionalizado, es decir no lineal, constituido por fragmentos de programación recuperados o sacados ad hoc de la red, incluso en streaming. Las entrevistas destacan cómo el uso no lineal se suele asociar a una competencia televisiva muy parcial, que desconoce las características de la oferta, las orientaciones de las emisoras y de los canales. Además de la idiosincrasia hacia el carácter generalista del broadcasting, la idea misma de la existencia de los horarios parece muy poco considerada: típico de las ideologías de la desintermediación, que Internet incorpora y promueve intrínsecamente, no intentan entrar en el pacto de confianza que un horario podría poner a disposición. Por lo tanto, la relación con la televisión quedaría opaca.

De entre todas las estrategias de relación con una ecología medial compleja como la contemporánea, la investigación ha detectado la de una multimedialidad distinta, capaz de reconocer y utilizar las especificidades de los medios. Por lo tanto, si Internet favorece o, mejor dicho, implica la presencia de fronteras menos netas y más fluidas entre los medios, entre géneros y contenidos, a este le corresponderían competencias y estrategias opuestas. Bajo esta perspectiva, además del enfoque temático a la televisión arriba mencionado, parece interesante y emblemático el uso de los periódicos.

La práctica de derivar la mediación institucionalizada de un medio, que se hace "parasitariamente" más frecuente en las entrañas de Internet, se repite en el ámbito de los periódicos, a través de la lectura de noticias aisladas, especialmente gracias a la costumbre de recibir estas noticias a través de la condivisión o el buscador de Internet: es decir, a través del filtro del algoritmo (Pariser, 2011; Sunstein ,2017). Sin embargo, el circuito cerrado del uso social de la televisión y de la prensa puede romperse: suele pasar en los casos en los que los jóvenes son más conscientes o toman conciencia de estos límites y desplazan el baricentro del consumo medial a través de las fuentes originales. Este desplazamiento suele ir acompañado del interés por la información en su autonomía, es decir de la superación del modelo del consumo de la información como producto secundario del sharing amistoso.

De esta manera, se definen las numerosas configuraciones personales de relación con los medios en las que la multimedialidad no es el resultado de la mezcla indistinta producida por algunas áreas de Internet, sino más bien aparece como el conjunto de competencias de uso y lectura basadas en la autonomía (relativa) del lenguaje, de los formatos y de las funciones de cada uno de los medios de comunicación - lo definiríamos, aunque de manera impropia, en clave analógica. Algunos de nuestros entrevistados no solo reconocen esta "repartición del trabajo" sino que también la experimentan y la "explotan" como recurso. En estos casos, la 
lectura del periódico, aunque en línea, tiende a recomponer la unicidad o, por lo menos, su identidad como producto cultural inspirado por tendencias políticas o por voluntades editoriales reconocibles, con las que establecer una relación.

Destacamos muchos casos de jóvenes que conciben algunos periódicos como punto de referencia para la adquisición y - aún más importante - para la interpretación de las noticias, tanto en el mundo de la televisión como en el de la prensa. Gracias también a un acceso "multi-plataforma", pueden beneficiar las producciones periodísticas all news de RAI y $S k y$, de La7, el Corriere della Sera y La Repubblica, hasta llegar a periódicos extranjeros como el Daily Telegraph: surgen motivos distintos, que mezclan inclinaciones político-culturales y preferencias de género y estilo. Esta relación privilegiada se enmarca en un contexto mixto, en el que evaluaciones y preferencias destacan claramente. A este propósito, Inta28 (21 años, de Perusa) representa un ejemplo de estas competencias cross-mediales: convencida de su carrera en relaciones internacionales y de su experiencia Erasmus, gestiona una interesante mezcla:

Leo el Internazionale. Estoy abonada al formato en papel, pero sigo también su página Facebook, o sea que las últimas noticias puede que las lea allí. (...) Repubblica online es insoportable, pero sigo Le Figaro y Le Monde.

Asimismo, podríamos definir cross-medial incluso la relación frecuente de Into 42 e Into 43 (estudiantes de Perusa, de 23 años) con la página Facebook de Mentana, director del telediario de $L a 7$, a partir de la preferencia por este periódico y por toda la programación de la información de esta emisora, que marca de manera significativa sus caminos de empoderamiento en la información política.

Se trata de dos maneras distintas de desplazar la confianza y asignar credibilidad. Por un lado, la confianza en los automatismos de la tecnología o del entorno social, que reflejan las preferencias personales y comunitarias; por el otro, la confianza en la mediación periodística, que se deriva de la autoridad percibida de los periodistas de algunos periódicos seleccionados. Por lo tanto, podemos observar que las dos configuraciones proponen dos distintas tipologías de desintermediación, que invierten de manera opuesta la función periodística: la primera expresa un camino que da por descontada (inercialmente más que voluntariamente) la posibilidad de derivar o no reforzar la función experta del trabajo periodístico; mientras que la segunda evita la mediación implicada por el networking digital, porque considerada engañosa. Esto significaría también formar o no parte de una determinada tipología de público, con sus prácticas y "pautas".

\section{ACTIVISMO DISCURSIVO Y LIDERAZGO DE OPINIÓN}

Para seguir con el tema de la confianza y de la influencia, y respetando la idea de observar un proceso constituido por experiencias, interacciones situadas y sobre todo construcción de relaciones, cabe mencionar las dinámicas de opinión dentro de los grupos-network a los que los jóvenes están conectados y dentro de la relación con los contenidos y los actores mediales.

Ante todo, es necesario pensar en los intercambios comunicativos entre pares como actividad que, por un lado, constituye un recurso dentro de su camino de identidad y, por otro, representa una herramienta útil para la interacción: plataformas personales para crear relaciones basadas en la afinidad y para comprometerse en público y defender ideas, temas o valores (Sunstein, 2009; Boccia, Artieri, et al. 2017). 
De entre todos los temas más atractivos, la presente investigación pone de relieve: la inmigración, la sexualidad y el sistema de derechos que gira alrededor de la afectividad y de las uniones entre personas; el trabajo y las perspectivas para los jóvenes, a menudo asociados al desarrollo local; los derechos humanos en perspectiva global; y, menos frecuente, la crítica a la política. En lugar de analizar su relevancia y articulación entre los entrevistados, en este artículo nos centraremos en analizar las ideas en acción, es decir en interacción.

Las conversaciones, más o menos focalizadas, a menudo referidas en las entrevistas, entre compañeros de mesa del comedor ("los amigos del comedor"), de la universidad, de piso, entre novios o entre amigos que "salen" juntos para ir a cenar o al cine, o que quedan con los grupos de su misma ciudad ("los amigos del pueblo"), especialmente los fines de semana. Otro ejemplo son las discusiones en las plataformas sociales, compuestas por las secuencias de los comentarios en los post, discusiones más expuestas a la mezcla de las no homogeneidades político-culturales. Por lo tanto, se trata de micro-foros de discusión, muy a menudo caracterizados por los contenidos de la comunicación mainstream, que, no obstante, no siempre consigue vincular los intercambios dentro de los mismos marcos.

Por lo que concierne las afinidades electivas, podemos presentar el caso de la amistad y del gran pacto intelectual entre Into41 e Into42, estudiantes de posgrado en Perusa, basados en un planteamiento liberal-progresista de la crítica a la sociedad, que se podría resumir en la fórmula jocosa "Italia está podrida", que agrupa en sus visiones el problema de la legalidad, la deriva populista, la falta de un reformismo eficaz, la persistencia de estereotipos. Comparten amplios y proficuos espacios de discusión con los compañeros de la universidad más listos intelectualmente. Comparten además la dificultad de dialogar y hacerse entender, precisamente a causa de estas posiciones, por sus amigos del pueblo, respectivamente, en pequeñas ciudades de Umbria y de Campania.

Sobresalen dos tipos de conversaciones que los jóvenes realizan dentro de los gruposnetwork de pares. El primer tipo se realiza entre grupos autoseleccionados por la comunión de intereses y de preferencias político-culturales, capaces de generar agendas de grupo o de pandas sociales, es decir prioridades temáticas fomentadas por el intercambio influyente entre afines, con el refuerzo mutuo de posiciones (Roessler 1999; Sunstein 2017).

Sin embargo, existen intercambios en contextos mucho más abiertos y no homogéneos, que ponen en juego y en "peligro" la posibilidad de ser influyentes.

Las entrevistas han mostrado que en estas segundas situaciones se crean micro-climas de opinión entre pares que empujan a los jóvenes a hacer frente a problemas de gestión de las relaciones. En este sentido, hemos detectado actitudes tanto de espiral del silencio, es decir de progresivo cierre autocensura (Noelle-Neumann, 1984; Boccia, Artieri, et al. 2017, 4.3), como de compromiso "paladín" (Sunstein, 2009). Los temas más divisorios, tal como la inmigración y la diversidad sexual, crean contraposiciones polarizantes.

En cuanto a la autocensura, Into2 podría ser un ejemplo: estudiante que ha dejado su ciudad para estudiar en Perusa, propenso a hablar de problemas socio-políticos con amigos en línea y offline y que, tras un triste episodio en Facebook (ofensas personales como respuesta a uno de sus post), ha decidido no volver a publicar comentarios acerca de algunos temas, para evitar de esta manera que "la gente se mofe de mí públicamente". También puede haber un silencio preventivo, como es el caso de Inta 7: "me encanta leer discusiones que se generan a partir de una noticia, pero casi nunca intervengo. Me parece inútil entrar en contraposición con otras personas".

Por otro lado, hay casos como el de Inta10, estudiante de Roma (voluntaria de Caritas), que interviene siempre que aparecen comentarios xenófobos o racistas en los post de Facebook. Lo hace incluso en su contra y observa decepcionada: "es como hablar con las 
paredes (...) tras un comentario con sentido te contestan con la imagen del duce y la frase "fuera los gitanos de nuestro país"“.

A causa de conflictos parecidos, y para evitar seguirle la corriente, se puede decidir borrar las "amistades". Inta12, de Roma, afirma:

Le quité mi amistad cuando escuché algunos comentarios fuera de tono acerca de los niños negros y de los judios, pero muy desagradables... Por eso decidí quitarle mi amistad, porque no me parecía correcto mantener una amistad con gente que opina de manera totalmente diferente a mí en cuanto a algunos temas. i¿Tú sabes que no comparto tu opinión y expresas tan claramente algunos conceptos que a mi me molestan mucho?! Y en ese caso decidí borrarlo de mi pandilla de amigos.

El liderazgo de opinión no se refleja solo en las interacciones entre pares. El mundo medial como mediador del espacio público, les puede ofrecer a los jóvenes algunas figuras de referencias moral y/o política, o incluso de atracción emotivo-afectiva, que pueden ejercer una función guía en la formación de actitudes. Las figuras más indicadas han sido Papa Francisco, Matteo Salvini (líder de Lega), Matteo Renzi (líder del Partido Democrático), el periodista Enrico Mentana y el escritor Roberto Saviano.

Además de las de los políticos, las argumentaciones más interesantes para el diseño de la presente investigación han sido las que muestran una influencia sobre temas de relevancia socio-política procedentes de actores que no pertenecen al ámbito de la política. Bajo esta perspectiva, Papa Francisco parece ser estimado transversalmente, tanto por los ateos como por los jóvenes que sienten pertenecer al mundo de la Iglesia. En cuanto a los primeros, Inta26, estudiante de servicio social en Perusa, afirma:

Para mí, una persona que tiene mucha voz, podría parecer obvio o banal, es el Papa. O sea este Papa es fantástico, para mi es tremendamente fantástico, es una persona muy vanguardista con respecto a todo el mundo cristiano-católico que lo rodea. Yo espero que dure, porque razona de una manera que, quizás pueda parecer atrevido definirlo así, pero un poco anticristiano.

Inta12, católica, comadrona y objetor de conciencia en cuanto al aborto, declara:

El Papa Francisco para mí es un gran Papa y una figura muy importante en el mundo. Además de ser el Papa, creo que es una de las figuras más famosas en todo el mundo. Y también porque es un Papa un poco innovador, o sea que podría modificar de alguna manera muchas cosas de la Iglesia que yo también pienso que deberían cambiar.

Por lo que concierne a Saviano, Inta10, estudiante de comunicaciones en Roma, señala:

Saviano es apreciable, además su página siempre está llena de cultura, cuenta un poco de todo, (...) de todas formas lo veo muy inteligente, intenta acercarse a los jóvenes. Ahora, sí, también ha participado en el programa Amici. Sí, él es un personaje que hay que estimar.

El periodista Mentana se cita entre los entrevistados que prefieren una información caracterizada por una independencia y una capacidad de seleccionar temas y puntos de vista no siempre alineados a corrientes mainstream. Además, se aprecia también por su activismo en Facebook (como Saviano) que lo hace más accesible en los entornos de la identidad digital.

En general, los personajes seleccionados por los entrevistados parecen contar con 
características comunes:

1) la capacidad reconocida de innovar;

2) ser percibidos como dialogantes con los jóvenes;

3) la cross-medialidad, es decir la capacidad de ser activos en más entornos mediales, adaptándose a las especificidades del medio (un ejemplo típico es la presencia en la televisión y en los medios sociales).

Todo esto parece como una manifestación más de una escena pública mediada por una especie de supermedium, que la dilata, la hace multiforme y, en términos públicos, accesible e inteligible a través de códigos y formas simbólicas múltiples y gracias a competencias de cruce cross-medial.

\section{IMPLICACIÓN Y MILITANCIA}

Cabe observar que parece proficuo leer el proceso de auto-realización de la ciudadanía en los caminos de construcción de la identidad de los jóvenes a través del desarrollo de las dinámicas de influencia. Con el mismo método, parece útil leer ahora las experiencias de militancia dentro de un contexto relacional que les confiere sentido. Empowerment personal, engagement intelectual y cívico, y compromiso socio-político aparecen conectados. De ahí que parezca necesario proponer elementos más pertinentes con las culturas cívicas (desde la perspectiva de Dahlgren), pero sobre todo desde la vertiente de las actividades que destaquen un engagement cívico y político "patente" (desde la perspectiva de Bennett). La investigación ha puesto de manifiesto numerosos casos de compromiso cívico o político en los que los caminos de la vida parecen estar involucrados con distintas intensidades pero de manera no marginal o improvisada, menos envolvente, a veces incluso provisional. El voluntariado social parece disponer de un espacio notable. Inta3 forma parte de una asociación en defensa de los animales que presidia un refugio para gatos y se ocupa de la adopción de animales abandonados. Inta10 e Inta12 han sido voluntarias en Caritas y en la Comunidad de sant'Egidio respectivamente, encargadas de la asistencia a los pobres. Into44 e Into45 forman parte activa en un grupo de adquisición de productos "kilómetro cero" en Perusa. Into 40 ha participado en la campaña de referéndum sobre Brexit como voluntario en un equipo de un exponente de Conservative Party; forma parte de un Comité para la mejora de las conexiones ferroviarias de Perusa y de Umbria. Intal frecuenta la asociación Omphalos, activa desde el ámbito de la diversidad sexual. Hay un determinado número de entrevistados relacionados con las actividades parroquiales, en los oratorios, sobre todo dirigidas a la catequesis y a la agrupación de más jóvenes; experiencia que se cruza, por ejemplo para Inta30, con la participación en dos ediciones de la Jornada Mundial de la Juventud. A este panorama tan variado se añaden cuatro casos de militancia auténtica.

Por razones de espacio, no podemos dar cabida de la manera en la que los entrevistados comprometidos en el voluntariado colocan en sus propias vidas estas experiencias; por lo tanto, hemos decidido presentar solo aquellos casos (menos numerosos) que clásicamente se podrían definir de militancia, en otras palabras de pertenencia orgánica a una organización política.

Abordemos ahora el tema de la militancia, centrándonos principalmente en las motivaciones y en las evaluaciones que, en las historias de los entrevistados, rodean estas experiencias de compromiso político, intentando enmarcar siempre cada acontecimiento en su contexto relacional. Las entrevistas que forman la presente investigación destacan tres casos de militancia de partido y uno de militancia de movimiento. Analicemos a 
continuación el perfil de los tres casos que las entrevistas nos han permitido poner mejor de manifiesto.

La comparación muestra claramente que la militancia política se rige por motivaciones, caminos de acceso y desarrollos muy distintos. No obstante, cabe subrayar que todos los casos seleccionados podrían enmarcarse en la clase-tipo de los funcionales-selectivos, con perfiles unidos por un bajo nivel de intensidad de la actividad en las redes sociales y por un elevado interés focalizado por la información, sin duda la de alta mediación periodística. Además, prevalentemente en las relaciones cortas la experiencia política adquiere sentido, aunque exista siempre un entramado entre relaciones directas y relaciones con los textos y los actores mediales. Este entramado relacional constituye claramente el contexto de su proyecto de identidad, en el sentido de un conjunto de los elementos a los que su identidad se enfrenta, desarrollándose y encontrando momentos de negociación por definición. En este sentido, empoderamiento personal e implicación cívico-política parecen avanzar juntos.

Into39, 25 años, estudiante de posgrado en Perusa, es militante de Gioventù Nazionale, la organización juvenil universitaria del partido de derecha Fratelli d'Italia. Su militancia es la parte "activa" de un ferviente interés por la política sobre todo extranjera (que se combina con su situación de estudiante de relaciones internacionales). Posee un conocimiento amplio y diversificado de periódicos y cotidianos, incluso de sus directores y periodistas especializados. Se trata de un caso que representa con fuerza el resultado de empowerment político y de engagement intelectual intenso por parte de la información política y de la experiencia de estudio. Es un aficionado del periodismo de calidad y de comentarios. Lee en línea distintas cabeceras de periódicos, realizando una selección por temas y autores. Prefiere ideas contracorriente; ama defender con ironía algunas posiciones que la intelectualidad liberal-progresista define como populistas.

En su historia, la experiencia de militancia empieza cuando algunos amigos, ya activos en la órbita de la derecha, le proponen constituir un Comité para el No al referéndum sobre la reforma constitucional de diciembre de 2016; por eso, ha trabajado en la campaña del referéndum como jefe de prensa y organizador de eventos, para luego decidir darse de alta. Un elemento importante ha sido verse reflejado en la experiencia de la chica que se convertiría luego en su novia en aquel entonces, ya militante del Partido Democrático. Comenta: "Me he dado cuenta de que es realmente bonito realizar actividades políticas... Aunque no comparta todo, he entendido que ensuciarse las manos hace bien". En cuanto al sentido de la experiencia política, señala: "Los chicos forman realmente la política con la P mayúscula, como los jóvenes democráticos que se lo curran mucho, sobre todo los del PD. La política, tal y como la entienden todos, las de los Palacios, es otra cosa, es política en otro sentido, cuando la política es una profesión, pero es solo eso".

En el período de la entrevista se trataba solo de una experiencia aún en sus inicios, pero es evidente que vive su militancia dentro de un contexto de "activismo" de inmersión en la lectura y en el estudio que parece aún más fuerte que la militancia misma, encontrando apoyo en los compañeros y en los amigos íntimos. Por este motivo, de entre todos los personajes públicos dignos de ser considerados como puntos de referencia, menciona a dos filósofos de izquierda, un docente de geopolítica, "es que voy a buscar lo que dicen", los que va a contracorriente; "a mí me gustan los tocapelotas... Digo: jolín, no me había fijado".

Por lo que concierne a la función de actualización de los hechos, que algunos siguen asignando a la televisión, afirma: "Si pienso: “¿qué habrá pasado en el mundo?”. Enciendo: ¡no! “¿Qué habrá pasado en el mundo?”. Mejor pincho”.

Declara que utilizando Facebook no podemos informarnos realmente. Privilegia las páginas de las agencias de prensa para las noticias de primera mano, para evitar lecturas 
demasiado oficiales o demasiado partesanas. En cuanto al Corriere della sera, dice que son "editorialuchas"; y de Repubblica: "Cuentan con buenos periodistas, no sería honesto... Pero si tengo que leer el elogio de la inmigración sin límites... ¡No!”. Sigue criticando: "Internazionale es realmente el pensamiento liberal en su máxima potencia... ¡¡He leído algunas noticias sobre Siria!!”. En positivo, afirma seguir el trabajo de algunos periodistas, "que leyendo sus noticias me he dado cuenta de que realmente saben mucho". A este propósito, habla de tres "firmas" de la política extranjera y, entre ellas, dos pertenecen a Il Giornale:

Me enfado con mis compañeros de la universidad: "Ah, es el periódico de Berlusconi, iqué asco!”. Sí, es el periódico de la familia Berlusconi, en cuanto a política interior es lo que es, pero en cuanto a la exterior no tiene nada menos, todo lo contrario tiene algo más que los otros.

En cuanto a su compromiso en las redes sociales, comenta: "¿Los amigos de Facebook? No sé... Unos 200-300... Alguien me definiría pringado”. Y de las discusiones en Facebook opina: "Hay fanáticos de esto, que se divierten haciendo comentarios sobre noticias en las páginas de los periódicos (...) yo no, hice de provocador en Facebook... Me divierto haciendo estas cosas, sobre todo porque espero que me contesten mal, y me divierto de esta manera". Incluso en Twitter: "Cuando hay debates todos contra uno, yo defiendo a ese uno". Pero prefiere hacerlo cara a cara: "Da gusto hacerlo en persona... Lo bonito con mis compañeros: no coincidimos pero nos burlamos, es afectuoso, ¿no crees?”.

Into24, estudiante de 24 años de Roma, licenciado en sociología, nos cuenta haber superado una crisis personal gracias al hecho de haber decidido tanto seguir su carrera universitaria, como haber buscado y encontrado trabajo en Protección Civil. Había que superar también la desilusión por la experiencia de militancia en la organización juvenil del Partito Democrático, que dejó hacía años: "Una crisis total; en este momento, si pienso en todo eso siento repulsa... A nivel humano ha sido una experiencia totalmente negativa". Básicamente ha faltado la posibilidad de "empatizar", pero no solo. Entre viejos militantes que no dejan encender la calefacción en la sección, jóvenes que hablan de los líderes, "me ha faltado mi espacio político", el de la propuesta y de la innovación. La describe críticamente, ante todo desde el punto de vista del ideal de un partido que, en cuanto reformista, debería estar en contacto con la sociedad:

¿Militancia cara a cara? Solo durante la campaña electoral básicamente... Depende del territorio... Hay muy poca participación por parte de la gente, porque la política se vive como un servicio que te tienen que dar (...) Te relacionas siempre con las mismas personas que están dentro como tú, te enajenas del mundo exterior y vives completamente separado de la realidad; si haces políticas y rompes con la realidad... No soy yo quien te dirá cómo termina el cuento.

Comenta estar decepcionado por la falta de reconocimiento, dentro del partido, de la educación y de las competencias que certifica en cambio el mundo exterior; sin embargo, su evaluación refleja sus instancias de auto-realización, es decir la percepción de su camino de la vida, subrayando incluso la paradoja de la relación entre los jóvenes y el partido, un partido que acaba cultivando una determinada tipología de jóvenes:

A menudo hablamos de chicos de 20-25 años que no tienen trabajo: este es el militante típico, por lo menos aqui en Roma... Chicos que no completan su carrera universitaria en el tiempo establecido, se licencian con 30 y pico años, que hoy en día tampoco podemos considerar jóvenes (...) Son pijos, se consideran mejores porque 
hacen política e viven separados de la realidad por conocimientos, por experiencias. Yo en cambio me doy cuenta de que cuenta muchísimo, conoces historias totalmente distintas de la tuya, muchas experiencias distintas de la tuya.

Analicemos ahora una experiencia muy apasionante de militancia en un movimiento. También Into44, estudiante de posgrado de relaciones internacionales, 23 años, es un "ex", pero no por renuncia como en el caso de Into24; sino por suspensión provisional de su presencia activa en la comunidad, una ciudad en la provincia de Oristano, en una zona en la que históricamente sigue operando un movimiento, de alcance regional, contra las bases militares instaladas en Cerdeña; un movimiento para la defensa del territorio y de las comunidades locales que también ha cruzado las instancias independentistas y las relativas al rechazo de los residuos nucleares (asunto presentado en un referéndum consultivo regional, muy movilizador, en 2011).

Su adhesión a los movimientos anti-bases empezó cuando era adolescente, en el fermento de una vida de "pueblo" muy politizada gracias a estos grupos, precisamente durante la época del referéndum sobre el nuclear. Siguió durante la etapa de sus estudios fuera de Cagliari, en donde, como estudiante de filosofía, se agregó a un grupo de amigos sensibles a las reivindicaciones radicales y antagonistas. Acerca del período universitario, afirma:

Participaba en algunas pandillas, algunos locales, club, ibamos a jugar al futbolín

o a beber, pero había una ideología de fondo; uno que acababa de nacer se llamaba

Club Independentista Hugo Chávez... Quedábamos siempre por ahi y esto me ha permitido mantener el interés por estos temas.

Además, ha organizado siempre con sus amigos (sigue utilizando "nosotros") un cinefórum en su pueblo, "trabajo participativo incluso para encuentros con otros cinefórum regionales". Asimismo, se ha desarrollado la experiencia contra las bases militares, gracias a la constitución de asociaciones de ciudadanos (como "Gettiamo le basi"), la recogida de firmas, la presión sobre los candidatos a los cargos locales. En cuanto a las campañas electorales locales y los intentos de partidos y candidatos de afirmarse por sus propios intereses:

Somos los que siempre están provocando (...) el hecho de formar parte de asociaciones te lleva a entender las dinámicas... A vivir más arraigado en la comunidad, a enfrentarse, a luchar más, a crear relaciones...

Desigualdades, historias de movimientos independentistas, derechos políticos constituyen el frame de sus intereses por el cine y los documentales televisivos, que recupera en streaming. Con los amigos de Perusa comparte el desinterés por la información mainstream y un frecuente intercambio sobre medioambiente y derechos relacionados. Sigue vigilando sobre el tema de las bases militares, buscando noticias en línea, que encuentra en periódicos locales y nacionales. Se compromete con Into45 en la organización de un grupo de adquisición en un barrio central de Perusa, pero no le parece tan importante como su anterior militancia.

Nos explica el problema de las bases militares como expropiación del territorio que la "comunidad" (otra palabra clave en su historia) considera haber sufrido. De hecho, la actividad política de Into44 se ha basado en el asociacionismo desde abajo y más concretamente en una estrecha red de relaciones locales que, en su historia, aparecen solo parcialmente compatibles con la comunicación en las plataformas: "todo nace del contacto humano directo". El sentido de pertenencia a la comunidad en la que se siente "arraigado", y 
el mismo amor por su tierra ("cuando pienso en el pueblo, en los lugares, me emociono") le permiten percibir ser distinto de los actuales compañeros de la universidad, mayormente porque no se siente proyectado hacia un futuro realizado en cualquiera otra parte del mundo, tema que aborda muy a menudo tanto con los nuevos como con los antiguos amigos. Analizando la situación actual y su proyecto de realizar una empresa zootécnica ecosostenible, afirma:

Es probable que casi nunca haya desarraigo... Cuando solicitaron la beca Erasmus, yo les dije en broma "yo ya estoy de Erasmus"... No quiero alejarme demasiado, es que tampoco puedo (...) Encontrarme con la única chica de Cerdeña, que además comparte tú mismo horizonte cultural, es que te sientes comprendido... Claro, estoy más ausente... (en futuro) será una presencia, de otra manera, pero será una presencia.

\section{CONCLUSIONES}

Con respecto a la utilidad heurística de los modelos de ciudadanía propuestos en los apartados iniciales, la presente investigación confirma que el modelo del self-actualizing citizen resulta eficaz si depurado de sus características normativas para dejar paso a la idea de procesualidad típica del concepto de culturas cívicas de Dahlgren. Traducir luego este concepto utilizando el de identidad, entendida en perspectiva hermenéutico-crítica, nos permitiría colocar provechosamente el aprendizaje y el ejercicio de la ciudadanía dentro de los caminos biográficos, tal y como se refleja en las entrevistas.

La investigación destaca la importancia de leer la relación de los jóvenes con la política y la ciudadanía desde sus puntos de vista y según sus razonamientos, que definiríamos en clave fenomenológica. El enfoque exploratorio a la realidad pública implica la puesta en marcha de recursos tanto individuales como relacionales, cuya disponibilidad y uso se incorporan de distinta manera: entre estos encontramos los sistemas de relaciones entre pares, la gestión del self en la web social, el uso de los distintos medios, la asignación de la confianza. Sin duda, la relacionalidad accesible en los distintos entornos comunicativos, online y offline, constituye el motor para la construcción del significado y del proyecto de identidad.

Un concepto como el del civic engagement parece adquirir por lo tanto un nuevo valor, en el mundo juvenil, si refleja una actitud de atención, de compromiso informativo, de exploración y de construcción de significados.

Nuestra investigación remarca que el proceso de orientación, es decir de decodificación y de comprensión, del espacio se traduce en un proceso de empoderamiento personal conectado de distintas maneras al de la identificación. De ahí que no sea conveniente mirar los éxitos "institucionales" del camino del proceso self-actualizing de la ciudadanía; más bien sería necesario desplazar la atención hacia el entramado de modalidades de apropiación.

En el uso de los medios sociales resaltan tres aspectos relevantes:

1) las costumbres y las preferencias evolucionan y se rediseñan a lo largo del tiempo biográfico;

2) en cuanto a la centralidad de Internet y a la cultura digital, notamos un cada vez más frecuente redimensionamiento y una reciente distancia de las plataformas sociales;

3) en la frecuencia de uso y en la extensión del networking de la plataforma observamos dos perfiles opuestos, según la orientación selectiva, que se consolida como una estrategia que prima la capacidad de "gobierno" activo de los medios de comunicación, pero en este caso digitales. 
En términos de consumo de la información, las habilidades selectivas resultan normalmente asociadas a otras dos competencias/estrategias: la multimedialidad y la crossmedialidad, ambas capaces de distinguir y emplear las específicas funciones de cada medio. La multimedialidad se traduce en una distinta asignación de la confianza en la función experta del periodismo con respecto a la implicada en la desintermediación propia del algoritmo.

Gestionar relaciones significa gestionar también las dinámicas de influencia: los jóvenes italianos entrevistados se encuentran muy a menudo a interactuar dentro de micro-foros de discusión entre pares (online y offline) en los que se definen contraposiciones polarizadas (mayoritariamente sobre inmigración). La investigación destaca dos estrategias posibles: la renuncia a la contienda, por el rechazo de las modalidades agresivas de los interlocutores, y el compromiso paladín en defensa de sus propias posiciones. Por lo tanto, de entre todas las formas significativas de participación en el espacio público, destacan el activismo informativo y el activismo discursivo, entre ellos estrechamente conectados: ambos, si presentes, manifiestan ser ocasión de empoderamiento personal y de implicación política.

En el ámbito de los discursos públicos accesibles en los entornos mediales, la investigación subraya (aunque de manera no generalizada) procesos de liderazgo de opinión construidos alrededor de figuras públicas tanto del ámbito político (líder de partido) como de ámbito no político (Papa Francisco, escritores y periodistas, entre otros). Las entrevistas destacan que, además de comunicar en los entornos más juveniles, se perciben como reputados e influyentes por sus capacidades de innovación y por el hecho de dialogar con el mundo juvenil.

Los temas políticos que resultan más atractivos son: la inmigración, los derechos relativos a la afectividad y a la sexualidad, el entorno, el trabajo, los derechos humanos en perspectiva global. Las agendas personales y de grupo amistoso resultan más sensibles a los eventos a los que el sistema de información italiano ha dado mayor espacio y énfasis a lo largo del período en el que se realizaron las entrevistas (sobre todo por lo que concierne los dos primeros temas). Sin embargo, destaca con fuerza la tendencia, sobre todo de los más entregados a la información, a concentrarse selectivamente en temas de interés que no coinciden con las prioridades de la información mainstream. Estos intereses focalizados generan conexiones que se basan en afinidades y que resultan relevantes por la definición de las preferencias informativas y político-culturales.

El amplio abanico de las distintas culturas cívicas definido por las dimensiones analizadas hasta ahora constituye el terreno de desarrollo para algunas formas de activismo dentro de organizaciones formales: entre estas últimas, la investigación ha destacado una preponderancia de casos de voluntariado social, con respecto a la mayor singularidad de la militancia de partido y de movimiento. En cuanto a estos últimos, hemos propuesto un análisis profundo de la manera en la que los jóvenes militantes elaboran y reconocen el sentido de su experiencia. Además de estar anclada en elecciones político-culturales, para todos adquiere un sentido dentro del entramado relacional y de sus dinámicas, por su capacidad de co-generar significados; y está conmensurada a las prioridades de crecimiento personal que se van formando en el "proyecto" del self.

Una última consideración acerca del método. Los resultados que acabamos de resumir se han concretizado en las historias que cada uno de los jóvenes italianos entrevistados ha presentado de sí mismos y en el razonamiento reflexivo de su propia experiencia, aspectos que ha sido objeto constante de esta investigación. A la hora de analizar todas las dimensiones definidas por el diseño, las entrevistas a fondo han intentado brindar una clave de lectura para la implicación cívica desde el punto de vista del actor social que lo vive y que 
lo reconstruye a nivel narrativo; lo hace dentro de un horizonte propio de valores y bajo una perspectiva cada vez más guiada por los recursos culturales disponibles en los entornos comunicativos en los que se mueve.

\section{BIBLIOGRAFÍA}

Addeo, F. y Montesperelli, P. (2007) Esperienze di analisi di interviste non direttive. Roma: Aracne.

Bartoletti, R. y Faccioli, F. (a cura di) (2013) Comunicazione e civic engagement. Media, spazi pubblici e nuovi processi di partecipazione. Milano: Franco Angeli

Benedicto, J. (2013) "The Political Cultures of Young People: An Uncertain and Unstable Combinatorial Logic". Journal of Youth Studies, 16(6): 712-729, DOI: 10.1080/13676261.2012.744812.

Benedicto, J. (2015) "Youth participation as meaningful citizenship experience: a challenge to improve democratic life", en European Commission Youth work and non-formal learning in Europe's education landscape Luxembourg: Publications Office of the European Union, pp. 217-229, en https://ec.europa.eu/epale/sites/epale/files/youth_work_and_non-formal_learning_in_europes_educa tion_landscape_and_the_call_for_a_shift_in_education.pdf [consulta 5/6/2018]

Benedicto, J. y Morán, M.L. (2003) (Eds.) Aprendiendo a ser ciudadanos. Madrid: INJUVE.

Benedicto, J. y Morán, M.L. (2007) "Becoming a Citizen. Analysing the Social Representations of Citizenship among Young People". European Societies, 9 (4): 601-622, http://dx.doi.org/10.1080/14616690701314085

Benedicto, J. y Morán, M.L. (2013) "De la integración adaptativa al bloqueo en tiempos de crisis. Preocupaciones y demandas de los jóvenes". En M.L. Morán (Ed.) Actores y demandas en España. Análisis de un inicio de siglo convulso. Madrid: Catarata.

Benedicto, J. y Morán, M.L. (2014) “¿Otra clase de politización? Representaciones de la vida colectiva y procesos de implicación cívica de los jóvenes en situación de desventaja”. Revista Internacional de Sociología. 72(2): 429-452, DOI: https://doi.org/10.3989/ris.2013.02.21

Bennett, L.W. (1998) "The UnCivic Culture: Communication, Identity, and the Rise of Lifestyle Politics", in Political Science and Politics, 31: 41-61.

Bennett, L.W. (Ed.) (2008a) Civic Life Online: Learning how Digital Media Can Engage Youth. Cambridge: MIT Press.

Bennett, L.W. (2008b) "Changing Citizenship in the Digital Age", in L.W. Bennett (Ed.) Civic Life Online: Learning How Digital Media Can Engage Youth. Cambridge: MIT Press, pp. 1-24.

Bolter, J.D. y Grusin, R.A. (1999) Remediation: Understanding New Media. Cambridge: MIT Press.

Boccia Artieri, G. (2016) Stati di connessione. Pubblici, cittadini e consumatori nella (Social) Network Society. Milano: Franco Angeli.

Boccia Artieri, G.; Gemini, L.; Pasquali, F.; Carlo, S.; Farci, M. y Pedroni, M. (2017) Fenomenologia dei social network. Milano: Guerini e Associati.

Bontempi, M. y Pocaterra, R. (Eds.) (2007) I figli del disincanto. Giovani e partecipazione politica in Europa. Milano: B. Mondadori.

.Caniglia, E. (2002) Identità, partecipazione antagonismo nella politica giovanile. Catanzaro: Rubbettino.

Chadwick, A. (2013) The Hybrid Media System: Politics and Power. New York: Oxford University Press.

Dahlgren, P. (2005) “The Internet, Public Spheres, and Political Communication: Dispersion and Deliberation, Political Communication, XXII (2): 147-162, Doi: 10.1080/10584600590933160.

Dahlgren, P. (2009) Media and Political Engagement. Citizens, Communication and Democracy. New York: Cambridge University Press.

Dahlgren, P. (2013) "Reinventare la partecipazione. Civic agency e mondo della rete, in R. Bartoletti y F. Faccioli (a cura di) Comunicazione e civic engagement. Media, spazi pubblici e nuovi processi di partecipazione. Milano: Franco Angeli pp. 17-37. 
Erikson E. (1959), Identity and the Life Cycle, New York, International Universities Press.

Erikson E. (1968) Identity, youth and crisis. New York: Norton.

Gamson, W.A. (1992) Talking Politics. Cambridge: Cambridge University Press.

Guantiett D. (ed.) (2000), Web.Studies. Rewiring Media Studies for the Digital Age. London: Arnold.

Harris, A. J.; Wynn, J. y Younes, S. (2010) "Beyond Apathetic or Activist Youth: 'Ordinary' Young People and Contemporary Forms of Participation". Young, 18(1): 9-32.

Livingstone, S. (1999) Making Sense of Television. The Psychology of Audience Interpretation. London: Routledge.

Losito, G. (2004) L' intervista nella ricerca sociale. Roma-Bari: Laterza.

Lovink, G. (2011) Networks Without a Cause: a Critique of Social Media. Cambridge: Polity Press.

Lum, C. M. K. (Ed.) (2006) Perspectives on Culture, Technology, and Communication: The Media Ecology Tradition. Cresskill, NJ: Hampton Press.

Marini, R. (Ed.) (2011) Altri Flussi. La comunicazione politica della società civile. Milano: Guerini.

Melucci, A. (1982) L'invenzione del presente. Bologna: Il Mulino.

Melucci, A. (1989) Sistema politico, partiti e movimenti sociali. Milano: Feltrinelli.

Montesperelli, P. (1998) L'intervista ermeneutica. Milano: Franco Angeli.

Morales, L. (2005) “¿Existe una crisis participativa? La evolución de la participación política y el asociacionismo en España”. Revista Española de Ciencia Política, 13: 51-87.

Morales, L. (2014) La consolidación de la 'matriz cultural' de la democracia en España (1982-1996)". En M. Pérez Ledesma e I. Sanz (Eds.) Historia de las culturas políticas en España, Vol. IV. Madrid: Institución Fernando el Católico.

Morán, M.L. y Benedicto, J. (2003) "Visiones de la ciudadanía entre los jóvenes españoles" Revista de Estudios de Juventud, edición especial 25 aniversario Constitución Española.

Morán, M.L. y Benedicto, J. (2016) Los jóvenes españoles entre la indignación y la desafección política. una interpretación desde las identidades ciudadanas, Proyecto Juventudes, Ultima Década, 44: 11-38.

Neuman, W.R.; Just M.R. y Crigler, A.N. (1992) Common Knowledge. News and the Construction of Political Meaning, Chicago, University of Chicago Press.

Noelle-Neumann, E. (1984) The Spiral of Silence: Public Opinion - Our Social Skin. Chicago: University of Chicago Press.

Pariser, E. (2011) The Filter Bubble. What the Internet is Hiding from You. New York: Penguin.

Prensky, M. (2001) "Digital Natives. Digital Immigrants". On the Horizon, 9 (5): 1-6.

Putnam, R.D. (2001) Bowling Alone: The Collapse and Revival of American Community. New York: Simon \& Schuster.

Roessler, P. (1999) "The Individual Agenda-Designing Process: How Interpersonal Communication, Egocentric Networks, and Mass Media Shape the Perception of Political Issues by Individuals", Communication Research, 26: 666-700, Doi: 10.1177/009365099026006002

Schudson, M. (1998) The Good Citizen: A History of American Civic Life. New York: Simon \& Schuster.

Schwartz, H. y Jacobs, J. (1979) Qualitataive Sociology. A Method to the Madness. New York: Simon $\&$ Schuster.

Serpieri, R. y Tota, A.L. (2018) Sociologia. Quali culure per altre educazioni possibili?. Milano: Franco Angeli.

Smith, N.; Lister, R.; Middleton, S. y Cox, L. (2005) "Young People as Real Citizens: Towards an Inclusionary Understanding of Citizenship", Journal of Youth Studies, 8 (4): 425-443.

Sorrentino, C. (2008) La società densa. Firenze: Le lettere.

Sunstein, C.R.. (2009) Going to Extremes: How Like Minds Unite and Divide. Oxford: Oxford University Press.

Sunstein, C.R. (2017) \#Republic: Divided Democracy in the Age of Social Media. Princeton: Princeton University Press.

Stella, R.; Riva, C.; Scarcelli, C.M. y Drusian, M. (2014) Sociologia dei new media. Novara: De Agostini.

Tapscott, D. (1998) Grown up digital. How the net generation is changing your world. New York: 
McGraw-Hill.

Thompson, J.B. (1995) The media and modernity. A social theory of media. Stanford: Stanford University Press.

Twenge, J. M. (2006) Generation me. Why today young Americans are more confident, assertive, entitled and more miserable than ever before. New York: Simon \& Shuster.

Watkins, S.C. (2009) The Young People and the Digital. What the Migration to Social-Network Sites, Games, and Anytime, Anywhere Media Means for Our Future. Boston: Beacon Press.

\section{Breve currículo:}

\section{Rolando Marini}

Profesor asociado en la Universidad para Extranjeros de Perugia, en el sector científico Sociología de los Procesos Culturales y Comunicativos. Es presidente de la licenciatura en Comunicación Internacional y Publicitaria y es miembro de la Junta Directiva de la Asociación Italiana de Sociología. Sus intereses de investigación y estudio se centran en la relación entre los medios y la política, en el periodismo, en el espacio público. Entre los volúmenes publicados, mencionamos: I media come arena elettorale (1997), Mass media e discussione pubblica. Le teorie dell'agenda setting (2006), Altri flussi. La comunicazione politica della società civile (2011), Media Logic. La logica dei media (2017). Financiación (2015-16) de la Región de Umbría (Comité Regional para las Comunicaciones) y financiación (2018) de la Universidad para Extranjeros de Perugia. 